\section{Enfermedad de Chagas en Jalisco, México}

Señor editor: en relación con el artículo "Conocimiento epidemiológico y situación actual de la enfermedad de Chagas en el estado de Jalisco, México", ponemos a su consideración los siguientes comentarios. La revisión que se hace en el artículo pone de manifiesto la importancia de un problema que requiere, como señalan sus autores, "de la participación más activa y concreta de los académicos y los investigadores" ${ }^{11}$ para la toma de decisiones en políticas de salud que al operarse por los servicios correspondientes, involucren a la población misma.
Con el ánimo de agregar algunos datos sobre la epidemiología histórica del padecimiento en el estado, consideramos importante señalar que en México el Dr. Luis Mazzotti, en 1937, describió la infección natural del Trypanosoma en el Triatoma, en varias áreas del país, entre ellas Jalisco. ${ }^{2}$

Además de los vectores señalados por el Dr. Lozano y cols., ${ }^{1}$ se han encontrado infectados por Tripanosoma cruzy, en nuestro estado:

\section{Cuadro I}

Casos infectados POR Tripanosoma CRUZI, REPORTADOS DURANTE I966I 974 SEGÚN MUNICIPIO

Municipio

Casos Porcentaje

De la Región Norte

\begin{tabular}{lll} 
Bolaños & I & 6.6 \\
\hline Colotlán & I & 6.6 \\
\hline Chimaltitan & I & 6.6 \\
\hline Santa María de los Ángeles & I & 6.6 \\
\hline San Martín de Bolaños & I & 6.6 \\
\hline Subtotal & 5 & 33
\end{tabular}

De la Región Ciénega

\begin{tabular}{lrr} 
Atotonilco & 2 & 13.3 \\
\hline Jamay & 1 & 6.6 \\
\hline Ocotlán & 2 & 13.3 \\
\hline Subtotal & 5 & 33
\end{tabular}

De la Región Centro

\begin{tabular}{lll} 
Acatlán de Juárez & I & 6.6 \\
\hline Guadalajara & I & 6.6 \\
\hline Tonalá & I & 6.6 \\
\hline Subtotal & 3 & 20
\end{tabular}

De la Región Altos

\begin{tabular}{lrr} 
Tepatitlán & I & 6.6 \\
\hline Subtotal & I & 7
\end{tabular}

De la Región Valles

\begin{tabular}{lrr} 
Ameca & I & 6.6 \\
\hline Subtotal & I & 7
\end{tabular}

Tota

$15 \quad 100$

Fuente: referencia 8
Cuadro II

Casos infectados por Tripanosoma CRUZI, REPORTADOS DURANTE | 966-| 974, SEGÚN ALTURA

Altura sobre el nivel del mar Casos Porcentaje

\begin{tabular}{lrr}
$900-999$ & 1 & 6 \\
\hline I 000-I 199 & 0 & 0 \\
\hline | 200-I 399 & 3 & 20 \\
\hline | 400-I 599 & 6 & 40 \\
\hline | 600-| 799 & 5 & 34 \\
\hline Total & 15 & 100
\end{tabular}

Fuente: referencia 8

Cuadro III

Casos infectados por Tripanosoma CRUZI, REPORTADOS DURANTE

| 966-1 974, SEGÚN NUMERO DE HABITANTES

Número de habitantes Casos Porcentaje

\begin{tabular}{lrr}
100 o menos & 5 & 33.5 \\
\hline $100-999$ & 5 & 33.5 \\
\hline $1000-4999$ & 2 & 13 \\
\hline 5000 y más & 3 & 20 \\
\hline Total & 15 & 100
\end{tabular}

Fuente: referencia 8

1. Triatoma braivlosky ${ }^{3}$

2. Triatoma bolivari ${ }^{4}$

3. Triatoma Dimidiata maculipennis ${ }^{5}$

4. Triatoma recurva ${ }^{6}$

5. Triatoma rubida ${ }^{6}$

Cabe señalar que en la región norte, el vector se conoce desde tiempos ancestrales; los indígenas Wixaricas (vulgarmente llamados huicholes) le denominan "chirrai" y durante los años sesenta la población de esos lugares relacionó la infestación de las casas por Triatomidos, con los rociamientos con DDT. De los hospederos con positividad a T cruzy también está reportado Scirus vulgaris. ${ }^{7}$ 


\section{Cuadro iv \\ CASOS INFECTADOS POR TRIPANOSOMA CRUZI, REPORTADOS DURANTE 1966-1974, SEGÚN AÑO Y SEMESTRE DE REPORTE}

\begin{tabular}{lccccc} 
Semestre de reporte & 1966 & 1967 & 1969 & 1973 & 1974 \\
Enero a junio & 0 & 1 & 0 & 0 & 1 \\
\hline Julio a diciembre & 2 & 0 & 7 & 4 & 0
\end{tabular}

Fuente: referencia 8

\section{CuadroV \\ Casos infectados por Tripanosoma CRUZI, REPORTADOS DURANTE 1966-1974, SEGÚN EDAD Y SEXO}

\begin{tabular}{|c|c|c|c|c|c|c|c|c|}
\hline \multirow[b]{2}{*}{ Edad en años } & \multicolumn{2}{|c|}{ Hombres } & \multicolumn{2}{|c|}{ Mujeres } & \multicolumn{2}{|c|}{ No Identificado } & \multicolumn{2}{|c|}{ Total } \\
\hline & Casos & $\%$ & Casos & $\%$ & Casos & $\%$ & Casos & $\%$ \\
\hline I o menos & I & 14 & & & & & I & 7 \\
\hline I a 4 & 2 & 29 & & & & & 2 & 13 \\
\hline 5 a 9 & I & 14 & 3 & 50 & I & 50 & 4 & 27 \\
\hline 10 a 19 & 2 & 29 & 3 & 50 & & & 5 & 33 \\
\hline 20 a 34 & 2 & 29 & & & & & 2 & 13 \\
\hline 60 & & & & & I & 50 & I & 7 \\
\hline Total & 8 & 53 & 6 & 40 & I & 7 & 15 & 100 \\
\hline
\end{tabular}

Ji cuadrada $<20$ años vs. $>19$ años $=7.7 \mathrm{p}<0.05$

Fuente: referencia 8

Tripanosoma cruzy como agente patógeno del hombre, se reconoce en Jalisco desde 1966, a raíz de los hallazgos de los "microscopistas" de la Jurisdicción XII de la Comisión Nacional para la Erradicación del Paludismo (CNEP), quienes habían acumulado cinco casos en los municipios jaliscienses de Chimaltitan y Santa María de los Ángeles y en los municipios de Tepechtlan y Jalpa del estado de Zacatecas, ubicados en los cañones de Bolaños, Tlaltenango y Juchipila; donde se venía haciendo pesquisa de paludismo desde 1962.

A partir de estos hallazgos se desarrollan las investigaciones de la enfermedad de Chagas en Jalisco, donde destacan los estudios del grupo universitario que dirige el Dr. Lozano. ${ }^{1}$

Los datos que a continuación se presentan se obtuvieron en 1974-75, cuando uno de los firmantes de esta carta dirigió el Departamento de Epidemiología de los Servicios Coordinados de Salud del estado.

Como se podrá observar, los datos sobre lugar, tiempo y persona en el periodo 1966-1974, orientan sobre el comportamiento epidemiológico de la enfermedad de Chagas en el Jalisco de esa época y pueden ayudar a contrastar la hipótesis sobre la influencia de los procesos de urbanización-industrialización y de globalización de la desigualdad, que ocurren en nuestro estado a partir de la segunda década del siglo pasado, en la incidencia y prevalencia de la Tripanosomiasis en nuestro medio.

Dr. Javier E. García de Alba G, Dra.Ana L. Salcedo Rocho Unidad de Investigación Social, Epidemiológica y de Servicios de Salud. IMSS-jalisco. javier91046@yahoo.com, analeticia_salcedorocha@yahoo.com.mx

\section{Referencias}

I. Lozano Kasten F, Magallón Gastélum E, Soto Gutiérrez Margarita, Kasten Monges M, Bosseno MF, Breniere SF. Conocimiento epidemiológico y situación actual de la enfermedad de Chagas en el estado de Jalisco, México. Salud Publica Mex 2008; 50: 508-5I5.

2. Mazotti L. Infección natural de Tripanosoma cruzi en Triatoma dimidiata (Crateille), en los estados de Yucatán, Campeche, Chiapas, Veracruz y Jalisco. Revista Mexicana de Medicina 1937; 17:283-286.

3. Martínez A, Carcavallo RU, Peláez D. Triatoma brailovsky: nueva especie de Triatominae de México. Chagas 1984: I: 39-42.

4. Carcavallo RU, Martínez A, Peláez D. Una nueva especie de Triatoma Laporte en México. Chagas 1987. 4: 477-477.

5. Pinto CF. Valor do rostro e antenas na caracteristisaçao dos géneros de tritomidos (Heminiptera, reduvidae) Bol Biol Sao paulo. 193I; 19:45-136.

6. Disponible en: www.unibio-unam.mx.chagmex/ DOCUMENTACION/mapas/tria-estado.htlm [Consultado el 8 de enero de 2009].

7. Tay J, Ortega M, Capin R. Estado actual de nuestros conocimientos sobre transmisores de la enfermedad de Chagas en Zacatecas y Jalisco, en México. Reporte de nuevas localidades infectadas. Rev Fac Med de México 1979; I5:22I-226. 8. García de Alba G JE, Salcedo Rocha AL, Márquez AM, Gómez RH. Notas sobre las primeras detecciones de la enfermedad de Chagas en el estado de Jalisco, México. Cirugía y Cirujanos 1996; 64: I I4-I I7. 